University of Nebraska - Lincoln

DigitalCommons@University of Nebraska - Lincoln

Faculty Publications, Department of Physics and Astronomy

Research Papers in Physics and Astronomy

2014

\title{
Quasi Monoenergetic and Tunable X-rays by Laser Compton Scattering from Laser Wakefield e-beam
}

\author{
Nathan D. Powers \\ University of Nebraska-Lincoln, ndp5@byu.edu \\ Isaac Ghebregziabher \\ University of Nebraska-Lincoln \\ Gregory V. Golovin \\ University of Nebraska-Lincoln, ggolovin2@unl.edu \\ Cheng Liu \\ University of Nebraska-Lincoln, cliu8@unl.edu \\ Shouyuan Chen \\ University of Nebraska-Lincoln, schen6@unl.edu
}

See next page for additional authors

Follow this and additional works at: https://digitalcommons.unl.edu/physicsfacpub

Powers, Nathan D.; Ghebregziabher, Isaac; Golovin, Gregory V.; Liu, Cheng; Chen, Shouyuan; Banerjee, Sudeep; Zhang, Jun; and Umstadter, Donald, "Quasi Monoenergetic and Tunable X-rays by Laser Compton Scattering from Laser Wakefield e-beam" (2014). Faculty Publications, Department of Physics and Astronomy. 157.

https://digitalcommons.unl.edu/physicsfacpub/157

This Article is brought to you for free and open access by the Research Papers in Physics and Astronomy at DigitalCommons@University of Nebraska - Lincoln. It has been accepted for inclusion in Faculty Publications, Department of Physics and Astronomy by an authorized administrator of DigitalCommons@University of Nebraska - Lincoln. 


\section{Authors}

Nathan D. Powers, Isaac Ghebregziabher, Gregory V. Golovin, Cheng Liu, Shouyuan Chen, Sudeep

Banerjee, Jun Zhang, and Donald Umstadter 
Year: 2014

Pages: $1-2$

\title{
Quasi Monoenergetic and Tunable X-rays by Laser Compton Scattering from Laser Wakefield $e$-beam
}

\author{
Nathan D. Powers, Isaac Ghebregziabher, Gregory Golovin, Cheng Liu, Shouyuan Chen, Sudeep Banerjee, \\ Jun Zhang, Donald Umstadter \\ Department of Physics and Astronomy, University of Nebraska-Lincoln, Lincoln, Nebraska 68588, USA \\ nathan.powers@huskers.unl.edu
}

\begin{abstract}
Quasi monoenergetic and tunable x-ray beams are reported by inverse-Compton scattering from laser wakefield accelerated electrons. The high peak brightness, ultrashort duration, and small size of the source make it uniquely suitable for many applications.

OCIS codes:(140.7090) Ultrafast lasers; (290.3200) Inverse scattering ; (350.5610) Radiation
\end{abstract}

Synchrotron x-ray sources play a critical role in the advancement of science and technology. However, the breadth of applications suitable for conventional radio-frequency-based synchrotron sources is limited by the size and cost of such devices. Several schemes, many of which are based on lasers[1], have been recently developed in order to create a compact and affordable source of $\mathrm{x}$-rays.

Inverse-Compton scattered x-ray beams produced from laser wakefield accelerated (LWFA) $e$-beams have been of particular interest because of the ultrafast duration, high peak brightness, and high energy of such sources. They also benefit from intrinsic synchronization of the $e$-beam and scattering laser because the LWFA drive laser pulse and scattering laser pulse originate from the same laser beam. While these all-optical sources have many benefits, the spectra of the $\mathrm{x}$-rays reported in previous experiments have been polychromatic[2-5]. We have now achieved $\mathrm{x}-$ rays with a quasi monoenergetic spectrum[6]. In addition, the central energy of the beams can be tuned over more than an order of magnitude from $60 \mathrm{keV}$ to above $1 \mathrm{MeV}$. These characteristics make the x-ray beam more useful for a broad range of applications.

The x-ray beams were produced using the 100-TW Diocles laser system at the Extreme Light Laboratory. The laser beam was split into two pulses by a beamsplitter. The first pulse, with 1.3-1.7 J of energy and 33 fs duration, was used to drive a laser wakefield produced using a dual-nozzle gas jet. The gas jet design limited the electroninjection region and allowed injection and acceleration to be independently controlled resulting in a tunable, quasimonoenergetic electron beam. The second pulse, with 0.13-0.17 J of energy and $150 \mathrm{fs}$ duration, was focused and scattered off of the accelerated electrons, acting as an optical undulator. The $e$-beam and scattering laser beam were overlapped $1.5 \mathrm{~mm}$ after the accelerator at an angle of $170^{\circ}$, as shown in

Fig. 1.

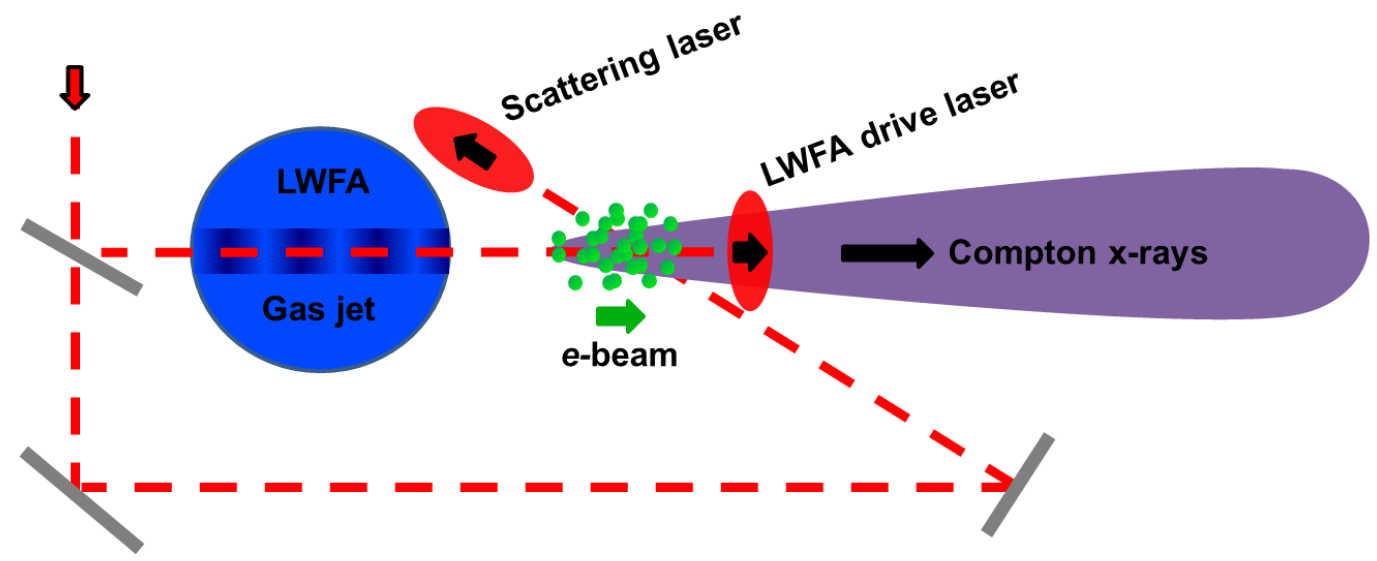

Fig. 1. Schematic configuration of Compton scattering from an LWFA-based $e$-beam

The x-ray spectral distribution, measured using Ross filter pairs, indicates a spectrum with a $66 \pm 7 \mathrm{keV}$ central energy and $\sim 50 \%$ FWHM energy spread. The numerically predicted results, simulated from the measured laser and $e$-beam characteristics, is shown in Fig. 2. These results are in good agreement with the measured results both in terms of central energy and energy spread, $64 \mathrm{keV}$ and 50\% FWHM, respectively. 
The x-ray central energy was tuned from $\sim 60 \mathrm{keV}-1 \mathrm{MeV}$ by tuning the $e$-beam energy. The central energy of the higher energy $\mathrm{x}$-rays was measured using a separate set of $\mathrm{x}$-ray transmission filters. Assuming an $\mathrm{x}$-ray energy distribution based on the measured $e$-beam spectrum, the $\mathrm{x}$-ray central energy was determined to follow a $4 \gamma^{2}$ scaling, where $\gamma$ is the relativistic Lorentz factor obtained from the $e$-beam central energy. The x-ray peak spectral brightness was $\sim 3 \times 10^{18} \mathrm{~s}^{-1} \mathrm{~mm}^{-2} \mathrm{mrad}^{-2}$ (per $0.1 \%$ bandwidth) for $70 \mathrm{keV}$ beams and $\sim 1 \times 10^{19} \mathrm{~s}^{-1} \mathrm{~mm}^{-2} \mathrm{mrad}^{-2}$ (per $0.1 \%$ bandwidth) for $1 \mathrm{MeV}$ beams.

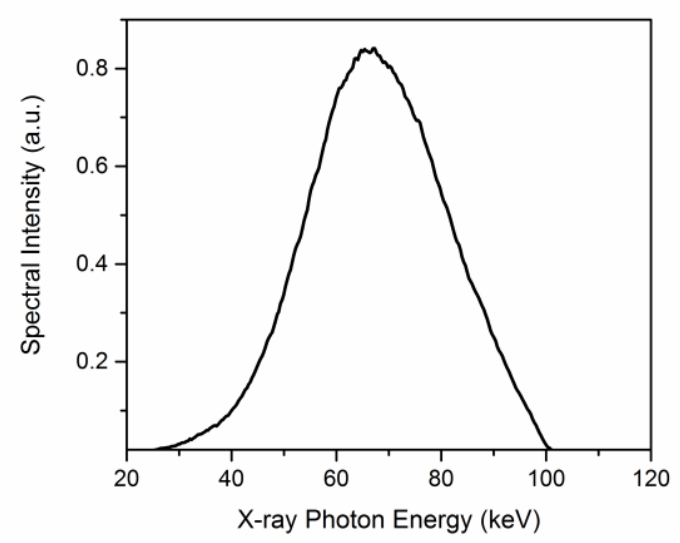

Fig. 2. X-ray spectral intensity simulated from measured LWFA $e$-beam

X-rays were produced for more than $93 \%$ of the laser shots with a photon number stable to within $60 \%$ of the average measured value, $1.7 \times 10^{6}$. The instability was primarily due to fluctuations in the $e$-beam charge. The $e$ beam energy spectrum was quasi monoenergetic over the entire tuning range. Based on measurements of the energy spectrum of the electron beam, $56 \%$ of the x-ray beams, over the entire range of tunability, had an energy spread of $\leq 50 \%$.

This proof of principle experiment opens up exciting possibilities for the future of x-ray light sources. For instance, we expect that this x-ray source will be useful for studying ultrafast structural dynamics due to the predicted femtosecond temporal duration. In addition, medical imaging and treatment will be possible with further improvements in the average brightness. Finally, if higher-energy electron beams are used[7], the x-ray source can be used for photonuclear studies.

[1] S. Corde, K. Ta Phuoc, G. Lambert, R. Fitour, V. Malka, A. Rousse, A. Beck, and E. Lefebvre, "Femtosecond x rays from laser-plasma accelerators," Rev. Mod. Phys. 85, 1-48 (2013).

[2] H. Schwoerer, B. Liesfeld, H. P. Schlenvoigt, K. U. Amthor, and R. Sauerbrey, "Thomson-backscattered x rays from laser-accelerated electrons," Phys. Rev. Lett. 96, 14802 (2006).

[3] Y. Mori, H. Kuwabara, K. Ishii, R. Hanayama, T. Kawashima, and Y. Kitagawa, "Head-On Inverse Compton Scattering X-rays with Energy beyond $10 \mathrm{keV}$ from Laser-Accelerated Quasi-Monoenergetic Electron Bunches," Appl. Phys. Express 5, 056401 (2012).

[4] K. Ta Phuoc, S. Corde, C. Thaury, V. Malka, A. Tafzi, J. P. Goddet, R. C. Shah, S. Sebban, and A. Rousse, “All-optical Compton gamma-ray source," Nat. Photonics 6, 308-311 (2012).

[5] S. Chen, N. D. Powers, I. Ghebregziabher, C. M. Maharjan, C. Liu, G. Golovin, S. Banerjee, J. Zhang, N. Cunningham, et al., "MeVEnergy X Rays from Inverse Compton Scattering with Laser-Wakefield Accelerated Electrons," Phys. Rev. Lett. 110, 155003 (2013).

[6] N. D. Powers, I. Ghebregziabher, G. Golovin, C. Liu, S. Chen, S. Banerjee, J. Zhang, and D. P. Umstadter, "Quasi-monoenergetic and tunable X-rays from a laser-driven Compton light source," Nat. Photonics 8, 28-31 (2014).

[7] S. Banerjee, N. D. Powers, V. Ramanathan, I. Ghebregziabher, K. J. Brown, C. M. Maharjan, S. Chen, A. Beck, E. Lefebvre, et al., "Generation of tunable, $100--800 \mathrm{MeV}$ quasi-monoenergetic electron beams from a laser-wakefield accelerator in the blowout regime," Phys. Plasmas 19, 56703 (2012). 